\title{
Further study of encoding and arousal factors in free recall of verbal and visual material'
}

\author{
JEFFREY R. SAMPSON, Department of \\ Computing Science, The University of \\ Alberta, Edmonton, Alberta, Canada
}

In an effort to extend earlier findings to additional conditions, 72 male $S s$ were presented 24 items, half as words and half as line drawings, with or without instructions to try to remember them. Free recall was tested immediately after presentation and/or 1 day later. Picture recall was superior to word recall in all conditions, extending the previous finding. Other, GSR-related, aspects of the earlier results were not confirmed. Double encoding of pictures, through spontaneous generation of verbal labels, still appeared a reasonable explanation for the superior memory; but the role of arousal factors was no longer clear.

Kaplan, Kaplan, \& Sampson (1968) found better immediate memory for figural than for verbal stimuli with Ss set to learn. In addition, mean GSR scores based on items presented as words predicted both word and picture recall, while GSRs based on the figural presentations did not. It was suggested that these GSR findings pointed to a verbal encoding for both words and pictures. Since pictures appeared to be coded visually as well, their double coding was offered as an explanation for the superior recall finding.

To test the validity of these results in a greater variety of conditions, notably in the absence of a learning set and with a relatively long delay before recall test, a second study was undertaken (in connection with the author's doctoral dissertation-Sampson, in press). To preserve comparability with the first study, identical stimuli, similar instructions, and comparable Ss were used. The major extensions of the earlier design were three: (1) the delayed recall session was advanced from 30 min after stimulus presentation to 1 day later; (2) many Ss participated in only one of the two recall sessions, instead of all being doubly tested; and (3) some Ss were not told in advance that recall would be involved (all Ss knew this in the earlier experiment).

$$
\text { METHOD }
$$

The Ss were 72 undergraduate males, whose participation partially fulfilled an introductory course requirement.

The stimuli consisted of 24 items: ARROW, BELL, BOAT, BOTTLE, CAT,
CIRCLE, FUNNEL, LAMP, PIPE, STAR, SUN, WINDOW, BIRD, BOOK, CANE, CUP, FISH, FLOWER, FORK, MOON, PENCIL, SQUARE, WATCH, and WHEEL. Half the Ss saw the first 12 of these as words (typed, capital letters) and the remainder as pictures (simple line drawings), while the situation was reversed for the other half. This division, combined with two orders of presentation, yielded four stimulus lists, which were balanced across treatment groups.

The stimuli were displayed individually on every third slide of a group of 73 slides, each of which was presented for $4 \mathrm{sec}$, with a negligible interslide interval. Except for an initial slide containing the word READY, every nonstimulus slide contained four differently colored dots, the colors of which were named aloud by the $S$.

The Ss wore zinc electrodes throughout all sessions. Skin resistance was recorded during the slide presentations and the first few minutes of each recall session. The modified Lykken electrodes are described in Kaplan \& Fisher (1964), and the skin resistance recording setup is described in Kaplan \& Hobart (1965).

All Ss were given a brief practice trial, to insure that they understood the instructions, which were to name the colors but not to respond to the words and pictures.

Two conditions were systematically varied, producing the six $12-\mathrm{S}$ treatment groups shown in Table 1 . The recall condition involved whether Ss had a single recall task or were doubly tested both immediately and at 1 day after stimulus presentation. The T1-T2 abbreviations thus distinguish the recall session at 1 day as the first or second test for that group of Ss. The set condition involved whether or not Ss were told, as part of the prestimulus instructions, that they would later be asked to remember the items. Nonset Ss were told only that it was important for them to pay careful attention to the items so that a meaningful measure of their skin resistance could be obtained. 2

The recall task consisted of giving the $S$ paper and pencil and asking him to reproduce as accurately as possible as many items as he could remember, in any order. The immediate session was begun approximately $30 \mathrm{sec}$ after the last stimulus item. For the 1-day test, the Ss involved returned during the same hour on the day after stimulus presentation; the I-only groups did not return at all.

A GSR score was computed for each $\mathrm{S}$ for each word and picture item. This was obtained by calculating the percentage decrements in skin resistance (a) from the maximum point in the $12-\mathrm{sec}$ interval following stimulus onset to the lowest value following it (in time) in the interval, and (b) from the minimum in the interval backward to the highest value preceding it; the difference of the two values in each case was divided by the larger to produce the percentage. The larger of the (a) and (b) percentages, which are equal unless the maximum in the interval follows the minimum, is then taken as the GSR on that item (the score being 0 if there is no decrement in the interval). This procedure produces a measure of the largest resistance decrement in the forward-time direction, ignoring local maxima, in the 12-sec interval beginning with stimulus onset and ending with the termination of the second color slide to follow (except for the last item, where only one color slide followed, leaving the last $4 \mathrm{sec}$ of the scoring interval blank). ${ }^{3}$

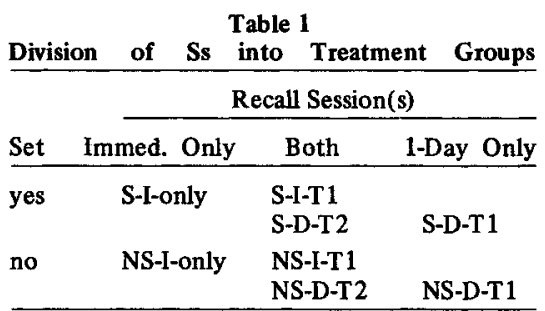

\section{RESULTS}

It was justifiable to combine immediate recall performances where the only difference between two groups was that one would return for a second experimental

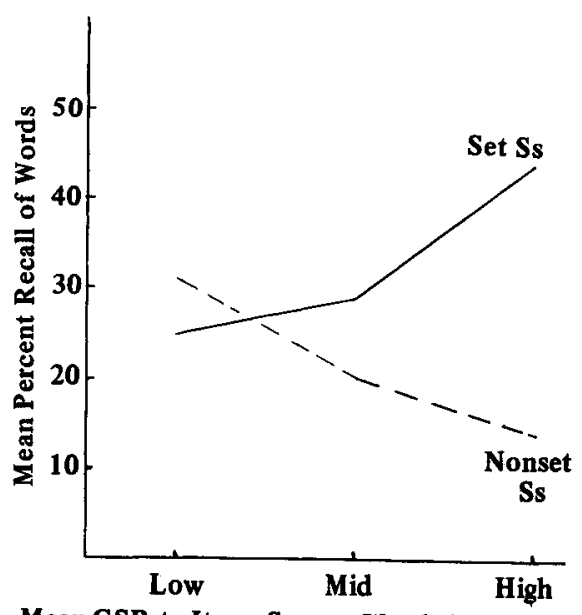

Mean GSR to Items Seen as Words by Set Ss

Fig. 1. Mean per cent immediate recall of words by set and nonset Ss as a function of grouping by mean GSR when the items are presented as words to set Ss. 
Table 2

Mean Recall Percentages

\begin{tabular}{|c|c|c|c|c|c|}
\hline Group & Words & Pictures & df & $\mathrm{F}^{*}$ & $\mathrm{p}<$ \\
\hline $\begin{array}{l}\text { S-I } \\
\text { NS-I }\end{array}$ & $\begin{array}{l}32.6 \\
21.6\end{array}$ & $\begin{array}{l}42.0 \\
33.0\end{array}$ & 1,46 & 13.91 & .005 \\
\hline $\begin{array}{l}\text { S-D-T2 } \\
\text { NS-D-T2 }\end{array}$ & $\begin{array}{l}23.6 \\
13.2\end{array}$ & $\begin{array}{l}34.7 \\
28.4\end{array}$ & 1,22 & 13.74 & .005 \\
\hline $\begin{array}{l}\text { S-D-T1 } \\
\text { NS-D-T1 }\end{array}$ & $\begin{array}{r}10.4 \\
6.3\end{array}$ & $\begin{array}{l}22.9 \\
12.5\end{array}$ & 1,22 & 9.94 & .005 \\
\hline
\end{tabular}

* F values for the word-picture factor in each of three two-way analyses of variance

session $(t=1.00$ for S-I-only and S-I-T1, $t=1.06$ for NS-I-only and NS-I-T $1, \mathrm{df}=22$ and $p>.30$, in both cases). Thus, Table 2 displays percentage recall data for just six cases, the four 1-day conditions and the two combined groups, S-I (set, immediate) and NS-I. The analyses of variance revealed no interactions between the word-picture differences and those attributable to set and/or time of test. The recall of pictures was greater than for words in all conditions.

Four independent rank orderings of the stimulus items with respect to the mean GSRs they produced were obtained, depending upon whether the item was viewed as a picture or word by set or nonset Ss. All Ss were used in the determination of these normative GSR values, which were then used to divide the stimuli into eight-item thirds. When immediate recall of pictures or words by set or nonset $\mathrm{Ss}$ is plotted as a function of these thirds, curves of the type analyzed by Kaplan, Kaplan, \& Sampson (1968) are obtained. It is thus possible to check their result in the case of set Ss, to extend the analysis to nonset Ss, and even to examine the recall of set Ss as a function of GSRs produced by nonset Ss, and vice versa. This last type of analysis could lead to some conclusions about the generality of the normative GSR rankings.

The present data revealed no significant functional relationships for recall of either type of item by either type of $S$ when the norma tive GSRs were based on pictures seen by set or by nonset Ss. GSRs based on words seen by nonset $S s$ also failed to predict recall of either words or pictures by either group. In the remaining case, GSRs produced to words by set Ss, although there was no significant functional relationship for picture recall by either group, the word-recall data yielded the significant and interactive relationships shown in Fig. 1 ( $F=5.40, d f=2,26, p<.01$ for recall by set Ss; $F=3.31, \mathrm{df}=2,46, \mathrm{p}<.05$ for recall by nonset $\mathrm{Ss} ; \mathrm{F}=8.45$, df $=2,92, \mathrm{p}<.001$ for the interaction).

\section{DISCUSSION}

There seems to be clear evidence that superior memory for the pictures is characteristic not only of immediate recall by Ss under a learning set (the conditions of the earlier study) but also of recall delayed a day (with or without a prior test of immediate recall), and (at either time) of recall by Ss not under a learning set. In the context of the double-encoding model, these findings suggest two hypotheses: (1) the attachment of verbal labels to visual stimuli is not strictly for mnemonic purposes-strong, fundamental cognitive habits appear to be involved;(2) such labels are not especially transient-they facilitate recall at least as long as 1 day later.

Findings of comparably superior free-recall performance for nonverbal stimuli(drawings or objects, contrasted with their names) by Ducharme \& Fraisse (1965), Lieberman \& Culpepper (1965), and Scott (1967) lend further credence to the view that double encoding of visual material may be a fairly ubiquitous phenomenon. In fact, Ducharme \& Fraisse (1965) found no improvement over memory for pictures when the names were added to the figural stimuli, which they interpreted as evidence for spontaneous verbalization when the picture was seen. 4

The situation with respect to arousal factors is considerably less clear. The word GSRs predicted recall only of words by set Ss, not of pictures as well, contrary to the findings of Kaplan, Kaplan, \& Sampson (1968). Furthermore, the opposite relation obtained for the recall of words by nonset Ss as a function of the word GSRs of the set Ss. This casts some doubt on the "normative" nature of the GSRs, since nonset Ss showed the same superior memory for figural stimuli as set Ss; it would now seem unwise to follow the suggestion of Kaplan, Kaplan, \& Sampson (1968) and use GSR values for objects viewed as words in research on recall of the objects as pictures. In addition, the import of the current findings for the double-encoding model is not clear; if there is any relationship between the GSR an item produces and its "encoding structure," as was suggested in the earlier study, it is obviously not a simple one. Further research would seem to be a prerequisite for further theoretical speculation in this realm.

\section{REFERENCES}

DUCHARME, R., \& FRAISSE, P. Étude génétique de la memorisation de mots et d'images. Canadian Journal of Psychology, 1965, 19, 253-261.

KAPLAN, S., \& FISHER, G. R. A modified design for the Lykken zinc electrodes. Psychophysiology, 1964, 1, 88-89.

KAPLAN, S., \& HOBART, J. L. New technique for recording skin resistance. American Joumal of Medical Electronics, 1965, 4, 117-120.

KAPLAN, S., KAPLAN, R., \& SAMPSON, J. R. Encoding and arousal factors in free recall of verbal and visual material. Psychonomic Science, 1968, 12, 73-74.

LIEBERMAN, L. R., \& CULPEPPER, J. T. Words vs. objects: Comparison of free verbal recall. Psychological Reports, 1965, 17, 983-988.

SAMPSON, J. R. A neural subassembly model of human learning and memory. Technical Report, Department of Computer \& Communication Sciences, University of Michigan, Ann Arbor, Michigan, in press.

SCOTT, K. G. Clustering with perceptual and symbolic stimuli in free recall. Journal of Verbal Learning \& Verbal Behavior, 1967, 6, 864-866. NOTES

1. This investigation was supported in part by Research Grant MH-11599 from the National Institute of Mental Health, United States Public Health Service, to Drs. S. and R. Kaplan, to whom the author is indebted for advice and assistance received while working in their laboratory at the University of Michigan, Ann Arbor, Mich.

2. Since it was not thought desirable to set $S s$ for recall when they would not have a memory task until the next day, the S-D-T1 Ss were actually given only an attention set by being told to watch for repetitions of stimulus items. This produced an improvement in recall relative to NS-D-T1, comparable to the differences between other set and nonset group pairs.

3. Kaplan, Kaplan, \& Sampson (1968) were in error in describing a somewhat different scoring procedure. The method described here was actually used for both experiments.

4. These findings were for adult Ss only; the children studied by Ducharme and Fraisse did no better on pictures alone than on words alone, but much better on the combined stimuli, perhaps suggesting that the "spontaneous" verbal encoding process develops with experience. 